\title{
Capacidad de concentración urinaria en lactantes desnutridos III parte
}

DRS.: JOSE CARDENAS I. ${ }^{\star *}$, FEDERICO PUGA C. *, GASTON ZILlERǓElo B. * JULIO BARROS S. ***.

En trabajos anteriores (1) hemos comprobado que el lactante desnutrido tiene limitada su capacidad renal para concentrar orina, y que dicha incapacidad es proporcional al grado de desnutrición. Por otra parte, cuando aumentamos el aporte exógeno de vasopresina (Pitresina), se observó una elevación manifiesta en la capacidad de concentración (2), obteniendo en lactantes con desnutrición severa una cifra de osmolaridad urinaria promedio estadísticamente igual a la obtenida en lactantes eutróficos. Los resultados antes mencionados nos indujeron a pensar que en el desnutrido existirían niveles bajos de HAD, o que la falla estaría a nivel del mecanismo efector de la acción hormonal, es decir, a nivel del sistema de la adenilciclasa tubular.

Sin embargo, la extensa información que reporta la literatura referente a alteraciones a nivel de la unidad "Túbulo-medular" ( 3,4 , $5,6,7,8,9,10)$ y la tendencia de los autores a considerar que el desnutrido severo es incapaz de construir un intersticio medular hipertónico, que permita el establecimiento de un gradiente osmolar efectivo, nos obliga a estudiar estos parámetros con mayor detenimiento, para así reforzar nuestra hipótesis de la falla a nivel del sistema hormonal.

MATERIAL Y METODO. Se formaron tres grupos de 5 lactantes cada uno, cuyas edades fluctuaban entre 6 meses y 2 años. Uno de estos grupos estaba formado por lactantes eutróficos en tanto que los dos restantes lo formaban desnutridos, con un déficit de peso comprendido entre el 40 y $60 \%$.

\footnotetext{
* Unidad de Nefrología. Hospital Luis Calvo Mackenna.

** Becado. Unidad de Nefrología. Hospital Luis Calvo MacBecado de Pediatría. Universidad Católica de Chile.
}

A los tres grupos se les sometió a pruebas de concentración urinaria (11), mediante deprivación acuosa de 12 horas, previo aporte de pitresina (Tanato de Pitresina, en suspensión oleosa, Parke Davis). Al final de la deprivación acuosa se practicó cateterismo vesical en forma aséptica y se conectó la sonda a tubos estériles. Simultáneamente con la muestra de orina obtenida por el cateterismo, se tomó muestras de sangre para determinar osmolaridad plasmática, así como nitrógeno ureico y electrolitos de sangre.

Inmediatamente después se les inició una infusión de Manitol de $3 \mathrm{~m}$ Osm/ $\mathrm{kgr}$ peso/ hora que duró 150 minutos. Durante la infusión de manitol se tomaron muestras de orina y sangre cada 30 minutos, con el objeto de medir osmolaridades, volumen urinario por minuto y electrolitos.

El aporte de pitresina fue de $0.08 \mathrm{U}$ vasopresoras por kilo de peso en los niños eutróficos y en uno de los grupos de desnutridos: a estos grupos los denominamos I y II respectivamente; al grupo restante, que se denominó III se le administró una dosis doble de pitresina (0.16 U V x kg de peso). El objeto de la sobrecarga con manitol fue el de determinar la curva de reabsorción tubular de agua y el $\mathrm{T}^{\mathrm{C}} \mathrm{H}_{2} \mathrm{O}$ máximo para cada niño, midiendo así la capacidad del riñón para establecer un intersticio medular hipertónico (12).

El Clearence Osmolar (Closm) y la reabsorción tubular de agua $\left(\mathrm{T}^{\mathrm{C}} \mathrm{H}_{2} \mathrm{O}\right)$ se determinó mediante las siguientes fórmulas:

\footnotetext{
Osmolaridad Urinaria

Closm: _ $\mathrm{X}$ Volumen Urinario minuto

Osmolaridad Plasmática

$\mathrm{T}_{\mathrm{C}_{2}} \mathrm{O}$ : Clearence Osmolar - Volumen Urinario minuto.
} 
Todos los valores obtenidos fueron estandarizados a $1.73 \mathrm{~m}^{2}$ de superficie.

Las osmolaridades, sea urinarias o plasmáticas, se determinaron mediante la técnica del punto de congelación en un osmómetro (Osmette, Precisión Osmometer).

Los electrolitos plasmáticos y urinarios se determinaron mediante un espectrofotómetro de llama. El nitrógeno uréico fue determinado por micrométodo descrito por O'Brien D., e Ibbott F. (13).

En todos los niños se descartó en forma previa al experimento, afecciones renales o neurológicas, realizándose además cultivos de orina al término de la experiencia.

\section{RESULTADOS}

GRUPO I: 5 niños eutróficos con un aporte de pitresina de $0.08 \mathrm{U}$ Vasopresoras por kgr de peso.

La osmolaridad urinaria promedio al término de la deprivación acuosa fue de 1.021 $\mathrm{m}$ Osm/1 con rangos entre 936 y $1.069 \mathrm{~m}$ $\mathrm{Osm} / 1$. La osmolaridad plasmática promedio fue de $307 \mathrm{~m}$ Osm/1 con valores que fluctuaban entre 290 y $317 \mathrm{~m}$ Osm/1.

Las cifras promedio de $\mathrm{Na}, \mathrm{K}$ y Nitrógeno Ureico plasmáticos al iniciar la sobrecarga con manitol fue de $147,8 \mathrm{mEq} / 1,4.62$ $\mathrm{mEq} / 1$ y $16.32 \mathrm{mg} \%$ respectivamente.

El Clearence osmolar máximo promedio, durante la experiencia fue de $6.42 \mathrm{cc}$ por minuto por $1.73 \mathrm{~m}^{2}$ con valores que fluctuaban entre 5.76 y $8.12 \mathrm{cc} \times \min \times 1.73 \mathrm{~m}^{2}$.

El T $\mathrm{T}^{\mathrm{C}} \mathrm{H}_{2} \mathrm{O}$ máximo promedio, fue de 3.96 cc $\mathrm{x}$ min $\mathrm{x} 1.73 \mathrm{~m}^{2}$ con rangos entre $3.60 \mathrm{y}$ $4.8 \mathrm{cc} \times \min \times 1.73 \mathrm{~m}^{2}$. Todos los valores de $\mathrm{T}^{\mathrm{C}} \mathrm{H}_{2} \mathrm{O}$ máximo en este grupo estuvieron por encima de la cifra límite reportada por Sommerschild: $3.2 \mathrm{cc}$ por minuto por $1.73 \mathrm{~m}^{2}$.

Ver figuras 1, 2, 3, 5 y 6 .

GRUPO II: 5 lactantes con desnutrición severa. Dosis de Pitresina $0.08 \mathrm{U}$ V x kgr de peso.

La osmolaridad urinaria promedio al iniciar el manitol fue de $743 \mathrm{~m}$ Osm/1 con rangos entre 503 y $905 \mathrm{~m} \mathrm{Osm/1.} \mathrm{La} \mathrm{osmolari-}$ dad plasmática promedio al inicio de la prueba fue de $307 \mathrm{~m}$ Osm/1 con rangos entre 300 y $312 \mathrm{~m} \mathrm{Osm/1.}$

Las cifras de $\mathrm{Na}, \mathrm{K}$ y Nitrógeno Ureico, promedio para este grupo, fue de $145 \mathrm{mEq} / 1$, $4.08 \mathrm{mEq} / 1$ y $13.08 \mathrm{mg} \%$ respectivamente.

El Clearence osmolar máximo promedio fue de $4.49 \mathrm{cc} \times \min \times 1.73 \mathrm{~m}^{2}$ con rangos entre 2.24 y $6.62 \mathrm{cc} \times \min \times 1.73 \mathrm{~m}^{2}$.

El $\mathrm{T}^{\mathrm{C}} \mathrm{H}_{2} \mathrm{O}$ promedio fue de $1.79 \mathrm{cc} \times \mathrm{min}$
FIGURA 1

Concentración urinaria al término de

la deprivación acuosa de 12 horas.

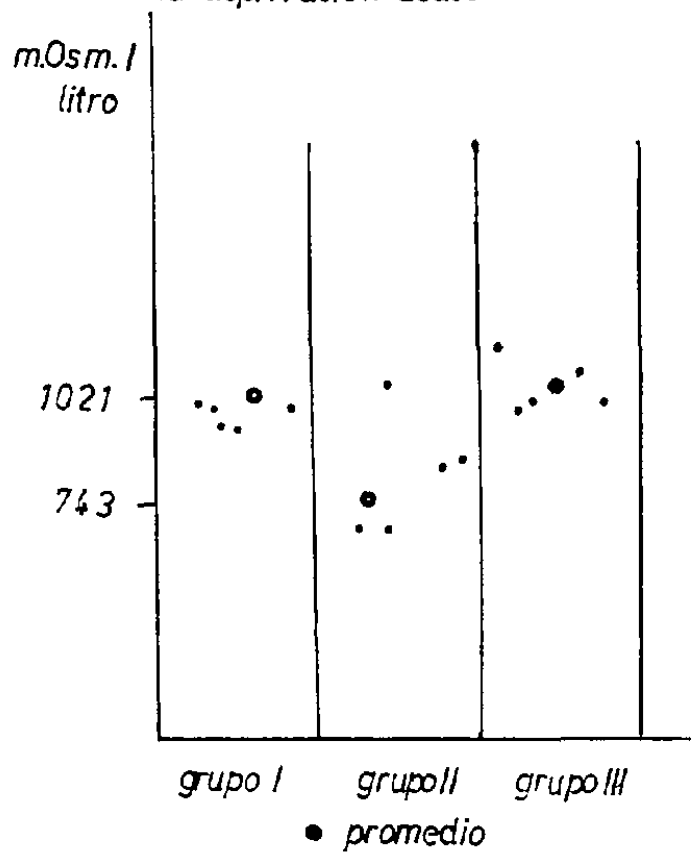

FIGURA 2

Clearance osmolar para cada lactante

por grupos y promedio

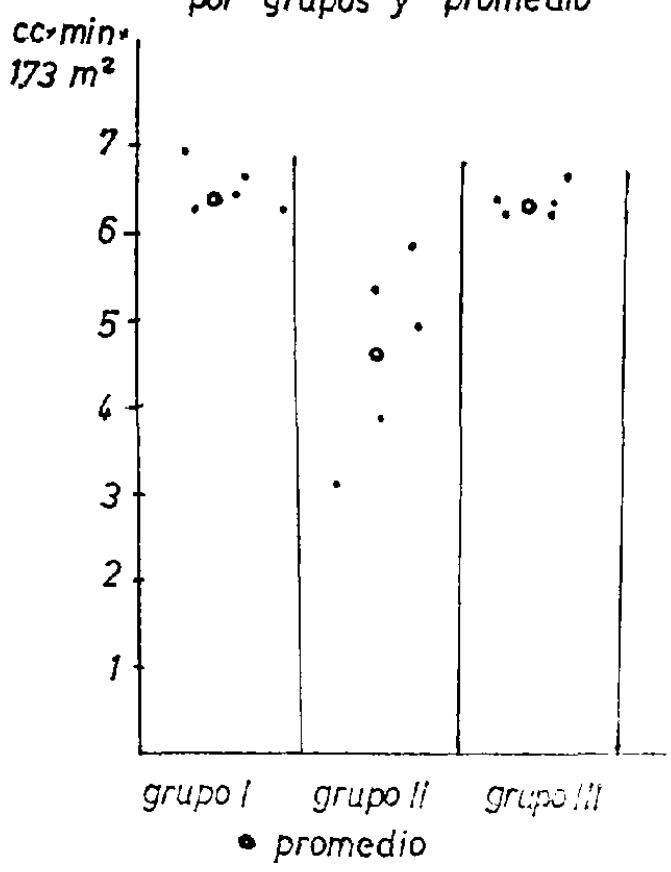


FIGURA 3

Tho maximo para cada lactante: grupos y promedio.

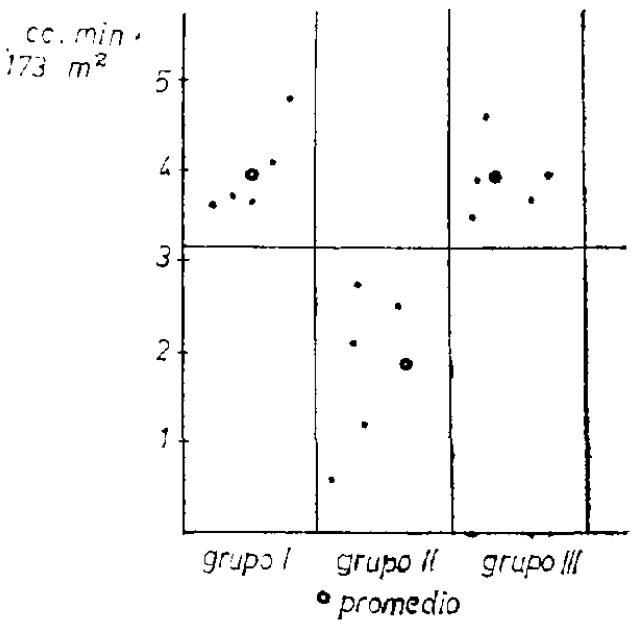

Figura No 4

Volumen urinario min promedio para cada grupo.

cc. $\times \min *$ $1.73 \mathrm{~m}^{2}$

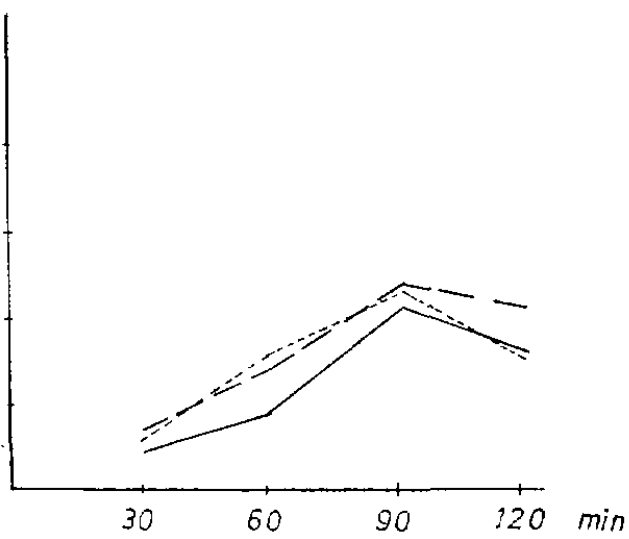

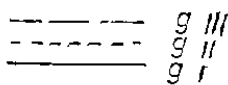

$\mathrm{x} 1.73 \mathrm{~m}^{2}$ con rangos entre 0.51 y $2.75 \mathrm{cc} \mathrm{x}$ $\min \times 1.73 \mathrm{~m}^{2}$.

En ninguno de los niños de este grupo se alcanzó a la cifra límite de $3.2 \mathrm{cc}$ por minuto por $1.73 \mathrm{~m}^{2}$.

Ver figuras 1, 2, 3, 5 y 6 .

GRUPO III : 5 lactantes con desnutrición severa. Dosis de Pitresina 0.16 U Vasopresoras por kgr de peso.

La osmolaridad urinaria promedio al término de la deprivación acuosa fue de 1.021 $\mathrm{m}$ Osm/1 con rangos entre 980 y $1.086 \mathrm{~m}$
Osm/1. La osmolaridad plasmática promedio en ese momento fue de $302 \mathrm{~m}$ Osm/1 con rangos entre 294 y $306 \mathrm{~m}$ Osm/1.

Las cifras de $\mathrm{Na}, \mathrm{K}$ y Nitrógeno Ureico promedio en plasma fueron de $145 \mathrm{mEq} / 1$, $4,5 \mathrm{mEq} / 1$ y $15.3 \mathrm{mgr} \%$ respectivamente.

El Clearence osmolar máximo promedio fue de $6.55 \mathrm{cc} \times \mathrm{min} \times 1.73 \mathrm{~m}^{2}$ con rangos entre 6.15 y $7.31 \mathrm{cc} \times \min \times 1.73 \mathrm{~m}^{2}$.

$\mathrm{El} \mathrm{T}^{\mathrm{C}} \mathrm{H}_{2} \mathrm{O}$ máximo promedio en este grupo fue de $3.94 \mathrm{cc} \times \min \times 1.73 \mathrm{~m}^{2}$ con rangos entre 3.50 y $4.67 \mathrm{cc} \times \min \times 1.73 \mathrm{~m}^{2}$.

En todos estos niños se alcanzó la cifra límite de $3.2 \mathrm{cc} \times \min \times 1.73 \mathrm{~m}^{2}$. Figuras 1, $2,3,5$ y 6 .

Figura $\mathbf{N}$ ? 5

Clearance osmolar promedio para cada grupo durante la sobrecarga con manitol

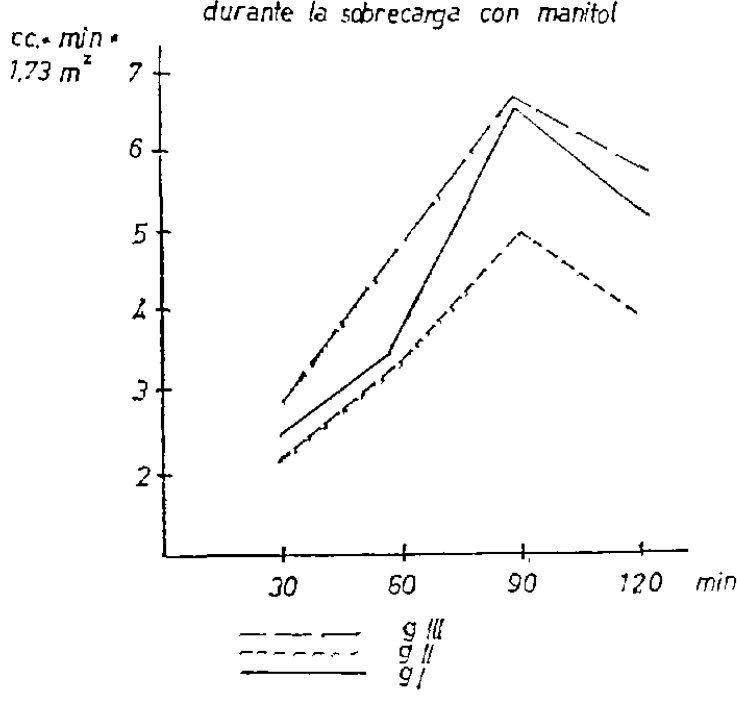

Figura $\mathbf{N} \div$

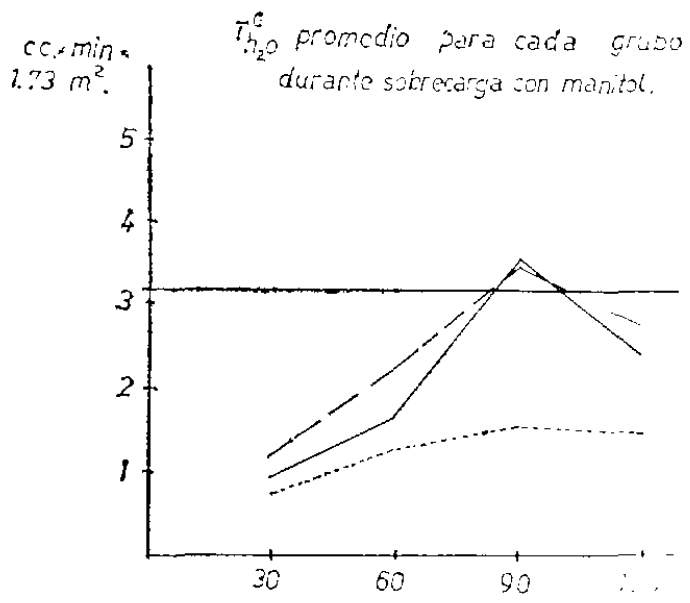


Los resultados de las concentraciones urinarias máximas al término de la deprivación acuosa son similares a los encontrados en las partes I y II de este trabajo sobre capacidad de concentración urinaria en el lactante desnutrido $(1,2)$.

Se obtuvo también las curvas promedios de volumen urinario minuto de estos tres grupos, así como las curvas de $\frac{\text { Closm }}{\text { Vol min }}$ y $\mathrm{T}^{\mathrm{C}} \mathrm{H}_{2} \mathrm{O}$

Vol min

para cada etapa del experimento.

Ver figuras 4,5 y 6 .

Los cultivos de orina practicados al finalizar cada experiencia resultaron negativos en todos los casos.

Comentario. La capacidad de concentración urinaria en el desnutrido, está afectada en función directa al grado de la desnutrición (1).

El mecanismo por el que el riñón realiza la función de concentrar la orina, con el objeto de garantizar la osmolaridad del medio interno, descansa en 2 factores esenciales:

I.- Un mecanismo de contracorriente efectivo, que permita el establecimiento de una gradiente osmolar entre el intersticio medular y el túbulo, capaz de movilizar agua.

II.- Un túbulo permeable al agua, lo que implica un sistema de adenil-ciclasa funcionante y niveles de HAD útiles.

El hecho de que la limitación a la capacidad de concentración urinaria que impone la desnutrición pueda ser modificada mediante el aporte exógeno de Vasopresina ( $\mathrm{Pi}$ tresina) en dosis eficiente (2), sugiere la posibilidad de que, en el desnutrido, sea el factor II el elemento determinante de la falla renal. Sin embargo, era imprescindible el estudio del factor I, a pesar de que ha sido objeto de innumerables trabajos cuyas conclusiones atribuyen a fallas en dicho factor, el papel principal del fenómeno observado en el desnutrido, ya que es lógico pensar que si falta la "fuerza motora" de atracción de agua, la reabsorción tubular no podría llevarse a cabo aún existiendo un túbulo permeabilizado. Además, el hecho conocido de que en el desnutrido hay una disminución franca en el flujo plasmático renal (F.P.R.) y en la velocidad de filtración glomerular, que condicionaría un bajo aporte de Sodio y Urea al intersticio medular, creando una gradiente osmolar deficitaria, avala la tesis sustentada por diversos autores referente a la etiopatogenia de esta falla renal $(8,9,13)$.

Sin embargo, la experiencia realizada por nosotros en lactantes desnutridos, a quienes estimulamos mediante deprivación acuosa y en quienes forzamos una diuresis osmolar con una sobrecarga de manitol, nos permitió medir en forma dinámica, la capacidad del riñón para establecer una médula hipertónica (12).

En los resultados obtenidos para determinar el $\mathrm{T}^{\mathrm{C}} \mathrm{H}_{2} \mathrm{O}$ máximo para cada lactante, así como los $\mathrm{T}^{\mathrm{C}} \mathrm{H}_{2} \mathrm{O}$ máximos promedios para cada grupo, observamos que existiendo incrementos en el volumen urinario minuto, estadísticamente similares en los tres grupos, el grupo de desnutridos a quienes se aplicó dosis dobles de pitresina, alcanzó en todos sus componentes valores de $\mathrm{T}^{\mathrm{C}} \mathrm{H}_{2} \mathrm{O}$ máximo similares a los alcanzados por los eutróficos con dosis simples, cifras que siempre estuvieron por encima del límite de normalidad señalado por Sommerschild. En cambio en el grupo conformado por lactantes desnutridos y a quienes se les aplicaron dosis simples de pitresina, en ningún caso alcanzó dicho límite. Variaciones similares se observaron como era de esperar en relación con los Clearence osmolares, que además de ser función del volumen urinario minuto, dependen del $\mathrm{T}^{\mathrm{c}} \mathrm{H}_{2} \mathrm{O}$, ya que al aumentar el $\mathrm{T}^{\mathrm{C}} \mathrm{H}_{2} \mathrm{O}$ se incrementa la osmolaridad urinaria, con un consiguiente incremento en el numerador de la fórmula para el Clearence osmolar.

$$
\text { Closm }: \frac{\text { Osmolaridad Urinaria }}{\text { Osmolaridad Plasmática }} \times \text { Vol. minuto. }
$$

También hay la disminución del denominador de dicha fracción al disminuir la osmolaridad plasmática a consecuencia de la retención de agua. Las parámetros antes analizados, sumados a la normalización mediante la sobrecarga con manitol de los factores que se supone alteran la capacidad de concentración, es decir, Flujo Plasmático Renal y Filtración glomerular dejan como gran variable, responsable del fenómeno, a la permeabilización tubular que es función de los niveles cuantitativos y cualitativos del sistema de la HAD y la conclusión evidente es que si bien el riñón del desnutrido en condiciones basales puede presentar una hipotonía a nivel del intersticio medular, lógico reflejo de la hipotonía generalizada, puede ante circunstancias que signifiquen un estímulo, construir una médula renal hipertó- 
nica, con una gradiente osmolar efectiva; pero la concentración urinaria final que es función de la reabsorción tubular de agua parece estar disminuida en el desnutrido debido a una falla en la permeabilización tubular.

Una aplicación práctica, de los resultados obtenidos en esta serie de trabajos en lactantes desnutridos, al descartar una falla renal permanente, no modificable a corto plazo por el médico, es que podrían modificarse los esquemas de rehidratación en el lactante desnutrido con deshidratación prolongada y rebelde a tratamiento, en los cuales con frecuencia desencadenamos una diuresis osmótica con pérdida progresiva y creciente de agua y electrolitos al no existir un incremento proporcional en el $\mathrm{T}^{\mathrm{C}} \mathrm{H}_{2} \mathrm{O}$ a causa de un túbulo poco permeable.

\section{RESUMEN}

Se practicó pruebas de concentración urinaria y se determinó el $\mathrm{T}^{c} \mathrm{H}_{2} \mathrm{O}$ máx. mediante una diuresis osmótica inducida por manitol en 3 grupos de lactantes; dos de estos grupos estaban formados por lactantes con desnutrición severa y a uno de estos grupos se le aplicó una dosis doble de Vasopresina exógena.

El grupo con dosis doble de pitresina $y$ el de lactantes eutróficos alcanzaron cifras de $\mathrm{T}^{\mathrm{C}} \mathrm{H}_{2} \mathrm{O}$ máx. superiores a las reportadas como limites de normalidad $\left(\mathrm{T}^{\mathrm{C}} \mathrm{H}_{2} \mathrm{O}\right.$ máx.: $3.2 \mathrm{cc} \times \min \times 1.73 \mathrm{~m}^{2}$ ). En el grupo de desnutridos con dosis simple de pitresina, ningún lactante alcanzó dicha cifra y se concluye que en el desnutrido severo, el factor determinante de la incapacidad para concentrar la orina, es la permeabilización tubular afectada por alteraciones cualitativas o cuantitativa en el sistema de la HAD.

\section{SUMMARY}

Urine concentration through $\max \mathrm{TCH}_{2} \mathrm{O}$ in osmotic diuresis induced by manitol, was measured in 3 groups of infants: 2 of them had severe malnutrition and double dose of Vasopresin was used in one of them.

The 3rd. group were normal infants.

The groups normal and malnourished with double dose of Vasopresin reached high levels of max $\mathrm{TCH}_{2} \mathrm{O}$, over normal reported data.

None of the malnourished infants with common dose of Vasopresin reached normal levels of concentration.

The incapacity to concentrate the urine could be due to a cualitative or cuantitative deffect of ADH. affecting the permeability of the renal tubule.

\section{REFERENCIAS}

1.- Cárdenas IJ., Puga C.F., Zilleruelo B.G., "Capacidad de Concentración Urinaria en lactantes desnutridos". I Parte. Rev. Chil. Ped. (En prensa).

2.- Cárdenas I.J., Puga CF., Zilleruelo B.G., "Capacidad de Concentración Urinaria en lactantes desnutridos". II Parte. Rev. Chil. Ped. (En prensa).

3.- Gordillo P.G., "Trastornos Renales en niños con desnutrición Avanzada". Bol. Méd. Hosp. Inf. Mex. 21: 699, 1964.

4.- Gordillo P.G., Soto R.A., Metcoff J. López E., "Intracelular composition and Homeostatics mecanisms in Severe infantil malnutrition. III Renal adjustement". Pediatrics 20: 303, 1957.

5.- Alleyne G.A., "The efects of severe Proteinecalorie malnutrition on the renal function of Jamaican Children". Pediatrics 39: 450, 1967.

6.- Edozien S., Phyllips EJ., "Partition of urine nitrogen in Kwashiorkor". Nature (London). 191: 47, 1961.

7.- Schwarts W.B., Relman A.S., "Metabolics and Renal Studies in cronic potasium deficiency resulting from overuses of laxative". J. Clin. Invest. 32: 228, 1953.

8.- Kerjel-Fronius C., Romhany G., Gati B., "Influences of depplettion of potassium, of sodium or of water on function and structure of the kidney". Pediatrics. 26: 939, 1960.

9.- Holliday M.A., Segar W.E., Bright N.H., "The effect of potassium deficiency on the kidney". Pediatrics. 26: 950, 1960.

10.- Levinsky N.G., Berliner R.W., "The role of Urea in the concentrating mechanism". J. Clin. Invest. 38: 741, 1959.

11.- Winberg $J$., "Determination of Renal concentrating capacity in infants without renal disease". Acta Paediat Scand. 48: 318, 1959.

12.- Sommerschild H.C., Grotte G., "Maximum Tubular reabsorcion of water". Acta Paediat. Scand. 59: 513, 1970.

13.- O'Brien D., Ibbott F. Laboratory Manual of Pediatric Micro and Ultramicro-Biochemical Techniques, Pág. 321, 1962. 